\title{
Visual Appearance and Its Fidelity Evaluation of Computer-Displayed Still Images
}

\author{
Masayuki IIZUKA, Makoto TAKEUCHI \\ Tokyo Institute of Polytechnics \\ Faculty of Engineering \\ 1583 Iiyama, Atsugi, Kanagawa 243-02, JAPAN \\ A part of this paper was presented at the 24th Joint Conference on Imaging Technology in Tokyo 1993, December.
}

Received January 4, 1994.

ABSTRACT

It seems that the visual appearance of a still image displayed in quasi-color is psychovisually different from that of in monochrome color. The visual impressions of computer-displayed images or reconstructed images are much affected by the various factors such as the size of image, pixel resolution, the number of gray level etc. It is pointed out that there is more or less a distinctive difference between the result of visual or subjective evaluations and the fidelity measure necessary for quantitatively estimating image quality.

The purpose of this paper is to quantitatively evaluate the difference between two images by means of some fidelity measures in connection with the visual appearance of computer-displayed still images. Moreover, a difference image representation is extensively used in order to carry out the intuitive discrimination between an original image and a reconstructed image, on condition that the size of block pixel is specified as constant in advance.

\section{Introduction}

Electronic display devices have been spread widely as the interface between people and computers. They are used to present information in the form of text and graphics. A personal computer can now display 8, 16, 256 , and 4096 colors, and a kind of workstation can display 16.8 million colors simultaneously.

A little unfamiliar phrase: "What you see is what you get.", often called WYSIWYG in the area of color industries becomes popular with a reproduction of multi or full color representation and a high fidelity technique on a color CRT device.

It seems that the visual appearance of a still image displayed in quasi-color is psychovisually different from that of in monochrome color. The intuitive impressions of computer-displayed images or reconstructed images are much affected by the various factors such as the size of image, pixel resolution, the number of gray level etc. It is pointed out that there is a difference between the result of visual or subjective evaluations and the quantitative fidelity measure necessary for estimating image quality. On the other side, the digital image compression and its application techniques have been studied from the viewpoint of an effective image transfer and/or a lot of digital image stocks. Recently, the JPEG (Joint Photographic Experts Group) recommended the standard method for effectively compress and decompress a digital image data.

In this study, some fidelity criteria are used in order to evaluate the image quality between two similar or different images. The visual impressions and appearance caused by the number of division of block-pixel are discussed in connection with the use of an adaptive spatial filter which is related to image compression procedure based on JPEG.

\section{Image Compression and Orthogonal Transform Techniques}

Image compression addresses the problem of reducing the amount of data required to represent a digital image $^{1)}$. The underlying basis of the reduction process is the removal of redundant data. From a mathematical 
viewpoint, this amounts to transforming a 2-D pixel array into a set of uncorrelated data statistically. We introduce the standard compression method recommended by JPEG in order to study the relationship between the visual appearance and its quantitative fidelity of computer-displayed still images.

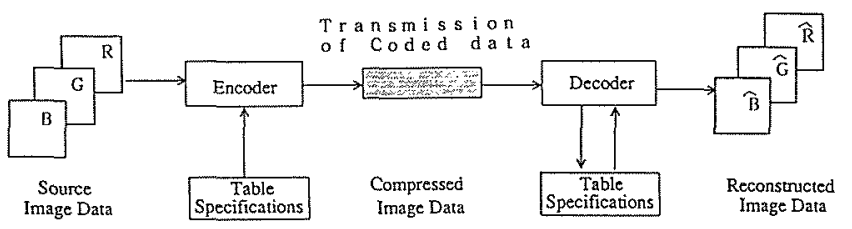

Fig. 1 Digital Image Compression Techniques by Means of JPEG

Fig. 1 shows a typical transform coding system for digital image compression techniques by means of JPEG. The compression system consists of two distinct structural blocks: an encoder and a decoder ${ }^{2}$. A set of RGB color image data may be transformed into the monochrome (or $\mathrm{Y}$ luminance component) data and the chrominance component data such as $\mathrm{CrCb}, \mathrm{IQ}, \mathrm{UV}$ etc. The $\mathrm{Y}$ luminance component data corresponding to the monochrome data may be computed simply using the following expression: $Y=0.299 R+0.213 G+0.114 B$, where the value of $R, G, B$ is specified as a kind of color level, for example, 0 to 15 , or 1 to 256 . On the other hand, the chromaticity: IQ, UV and $\mathrm{CrCb}$ may be obtained from a linear combination of R.G.B color components. For example, the YIQ system is the color primary system adopted by the NTSC (National Television Standards Committee) for color television broadcasting. Its main purpose is to exploit certain characteristics of the human visual system to maximize the use of a fixed bandwidth and to provide compatibility with black-and-white television. The YUV color space is the PAL (Phase Alternating Line) transmission standard mainly used in Western Europe. In this system the weighting coefficients used for the $Y$ component are the same as the NTSC system. The $\mathrm{YCrCb}$ system is almost equivalent to the YUV system, and has been developed for JPEG algorithm.

See a few books and a technical report at footnote for further information. ${ }^{\dagger}$

Fig. 2 shows a simplified and modified computation procedure based on the JPEG. It should be noted that an

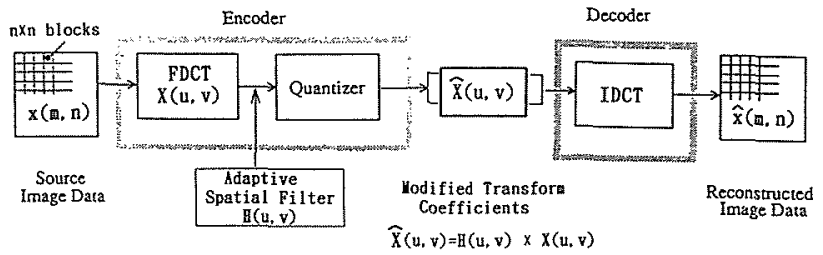

Fig. 2 Simplified Process for Image Compression by Means of Adaptive Spatial Filter

idea of the adaptive spatial filter in place of a quantization table is used in order to effectively quantize and truncate the transform coefficients regardless of the size of various block pixels. Transform coding techniques for image compression are omitted because of the intuitive and/or quantitative evaluation between two similar or different images.

An $\mathrm{N} \times \mathrm{N}$ original or input image data is subdivided into an $\mathrm{n} \times \mathrm{n}$ block data. Each block data is transformed using orthogonal transform techniques. The goal of the transformation process is to decorrelate the pixels of each subblock or pack a lots of information into the smallest number of transform coefficients. The main aspects of JPEG are to divide an original image into a small block pixel in order to decrease the computation time of an orthogonal transform technique such as DCT (discrete cosine tansform).

For 2-D arrays, the forward and inverse orthogonal transforms are formulated by the following matrix expressions.

$$
\begin{aligned}
& {[\mathrm{X}]=\frac{1}{\sqrt{\mathrm{MN}}}\left[\mathrm{T}_{1}\right] \cdot[\mathrm{x}] \cdot\left[\mathrm{T}_{2}\right]^{\mathrm{t}}} \\
& {[\hat{\mathrm{X}}]=\frac{1}{\sqrt{\mathrm{MN}}}\left[\mathrm{T}_{1}\right]^{\mathrm{t}} \cdot[\hat{\mathrm{X}}] \cdot\left[\mathrm{T}_{2}\right]}
\end{aligned}
$$

where, $[X]:$ transiorm coefficient

$[\hat{\mathrm{X}}]$ : modified transform coefficient

$[\mathrm{x}]$ : original image data

$[\hat{x}]$ : reconstructed (or modified) image data

$\left[T_{1}\right]$ : transform matrix for low direction

$\left[T_{2}\right]$ : transform matrix for column direction

$t \quad$ : symbol of transposed matrix

$M ; N$ : size of image (number of pixel)

+ Alan Watt: "Fundamentals of Three-Dimensional Computer Graphics", (1989) Addison-Wesley

David Travis: "Effective Color Displays, Theory and Practice", (1991) Academic Press; Reference (4)

Toshiaki Endo: International Standard Codeing System of Color Still Inage, -JPEG Algorithm-, Interface, No.175 (1991) pp. 160-182 [in Japanese] 
For example, the transform matrix for DCT may be written as follows.

$$
\begin{gathered}
{[\mathrm{T}]=[\sqrt{2} \cdot \cos (2 \mathrm{~m}+1) \cdot \mathrm{u} \cdot \pi / 2 \mathrm{M}]} \\
\mathrm{m} ; \mathrm{u}=0,1,2,3, \ldots \ldots, \mathrm{M}-1
\end{gathered}
$$

where, M: number of $\mathrm{m}$ (or $\mathrm{n}$ ) direction pixel

Several 2-D orthogonal transforms including the Fourier, Hadamard, sine etc. can be computed using the above two steps in the same way. In recent years, the discrete cosine transform has become the method of choice for standard image data compression.

The basis vectors in DCT resemble closely the basis vectors in Karhunen-Loeve transform (KLT) which has the best energy compaction property for smoothly varying the image data ${ }^{3)}$.

Discrete Fourier transform (DFT) is sometimes used for the digital image compression. The elements of the Mth basis vector of this transform for a matrix size $M$ is defined as $[T]=[\exp \{-\mathrm{j} 2 \cdot \pi \mathrm{mu} / \mathrm{M}\}]$, where, $\mathrm{j}=\sqrt{-1}$ and $\mathrm{m} ; \mathrm{u}=0,1,2, \ldots ., \mathrm{M}-1$.

The following expressions are the idealized mathematical definitions of the $N \times N$ FDCT and $N \times N$ IDCT which are recommended by JPEG ${ }^{4)}$.

$$
\begin{aligned}
X(u, v)= & (2 / N) C(u) C(v)\left[\sum_{m} \sum_{n} x(m, n)\right. \\
& \left.\cos \left\{\frac{(2 m+1) u \pi}{2 N}\right\} \cos \left\{\frac{(2 n+1) v \pi}{2 N}\right\}\right] \\
\hat{x}(m, n)= & (2 / N) \cdot\left[\sum_{u} \sum_{v} C(u) C(v) \hat{X}(u, v)\right. \\
& \left.\cos \left\{\frac{(2 m+1) u \pi}{2 N}\right\} \cos \left\{\frac{(2 n+1) v \pi}{2 N}\right\}\right]
\end{aligned}
$$

where, $\hat{X}(u, v)=H(u, v) \times X(u, v)$

$H(u, v)$ : adaptive spatial filter

$$
\begin{array}{ll}
C(u) ; C(v)=\sqrt{1 / 2} & \text { for } u ; v=0 \\
C(u) ; C(v)=1 & \text { otherwise }(u ; v=1,2, \ldots, \\
& \text { N-1) }
\end{array}
$$

The total energy in both domains: $x(m, n)$ and $X(u, v)$ or $\hat{X}(m, n)$ and $\hat{X}(u, v)$ should be the same because of orthogonality, therefore no energy is either lost or carried redundantly in the case of orthogonal transform techniques.

Digital image compression is achieved during the quantization of the transform coefficients, i.e., by a use of the adaptive spatial filter. In general, reconstructed image data $\hat{x}(m, n)$ may or may not be an exact replica of source image data $x(m, n)$. If it is, the system is error free or information preserving, and is called lossless compression. If it is not, some level of distortion is present in the reconstructed image, and the system is called lossy compression. In this case, the visual appearance of the reconstructed image with a noise such as block distortion differs from that of the original image.

Fig. 3 shows the typical three kind of divisions of the spatial frequency domain for an adaptive spatial filter. According to each division domain, for example, [a], [b], [cl, a weight value must be specified for alter and/or modify the value of transform coefficients in advance. A value of weight is specified as 1 in the $D C$ component and low spatial frequency domain [a]. The value of weight in the middle spatial frequency domain is specified as 0.5
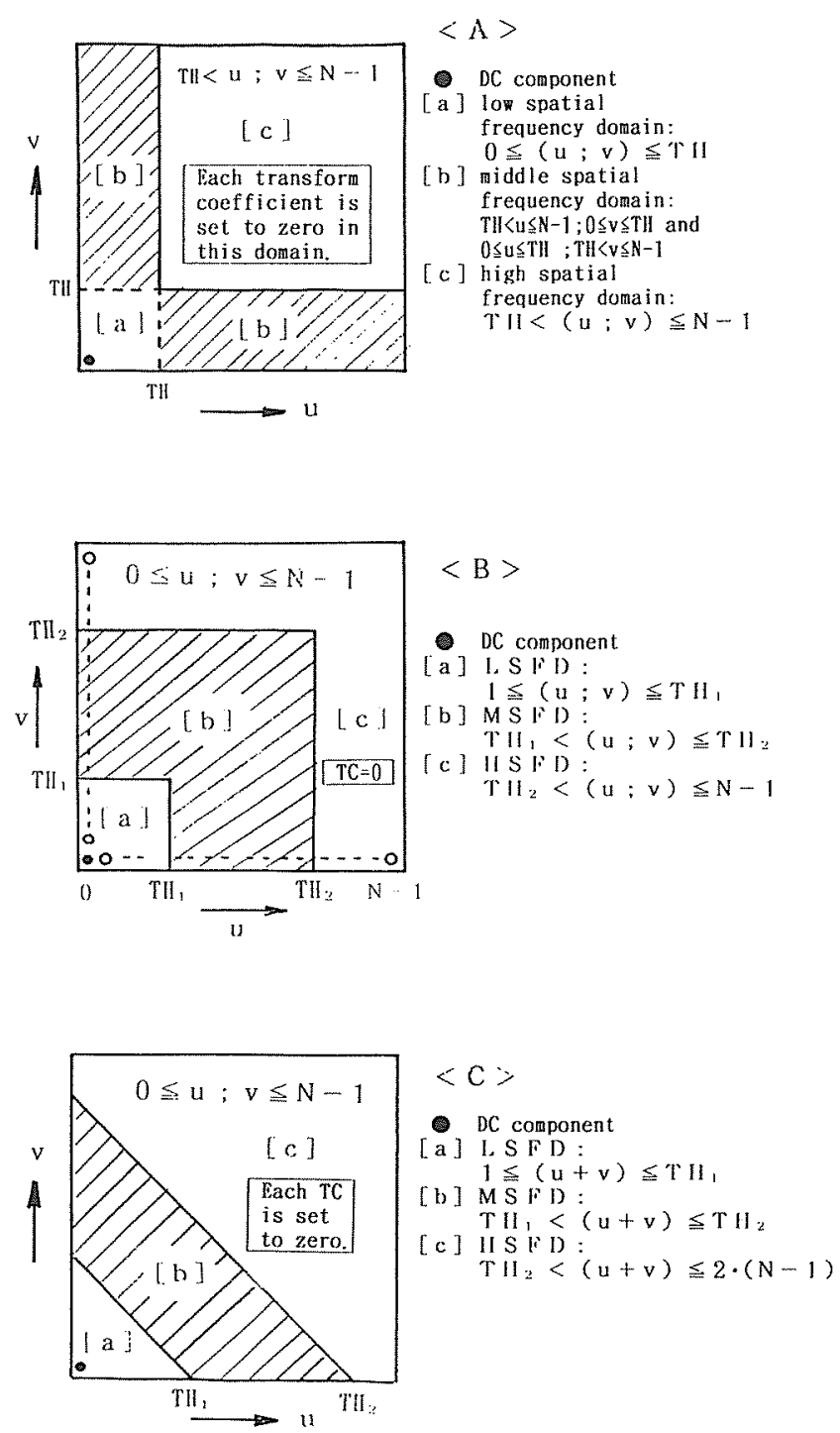

Fig. 3 Division of Spatial Frequency Domain 
for convenience. In the high spatial frequency domain, each value of weight is specified as 0 , because the significant value of transform coefficients is concentrated in the DC and low spatial frequency domains. As a result, the total number of modified and transform coefficient $\mathrm{X}(\mathrm{u}$, $v)$ is fairly reduced in contrast with that of original transform coefficient $X(u, v)$. In this case, a quantization table recommended by JPEG is unnecessary from the practical viewpoint of digital image compression regardless of the specified number of block pixel.

An adaptive spatial filter may be approximately simulated by applying a monotonously decreasing function of two variables $\mathrm{H}(\mathrm{u}, \mathrm{v})$. For example, the following function is useful for modify transform coefficients.

$$
\begin{aligned}
H(u, v) & =H(u) \times H(v) \\
& =\{1 /(1+k 1) \cdot(k 1+\cos [u /(N-1) \cdot \pi])\} \\
& \times\{1 /(1+k 2) \cdot(k 2+\cos [v /(N-1) \cdot \pi])\}
\end{aligned}
$$

where, $\mathrm{k} 1 ; \mathrm{k} 2=1,2,3$

Fig. 4 demonstrates a 3-D representation of the adaptive spatial filter which is defined by Eq. (4) in the case of the combination of two parameters: $\mathrm{k} 1=\mathrm{k} 2=1$ and $\mathrm{k} 1=$

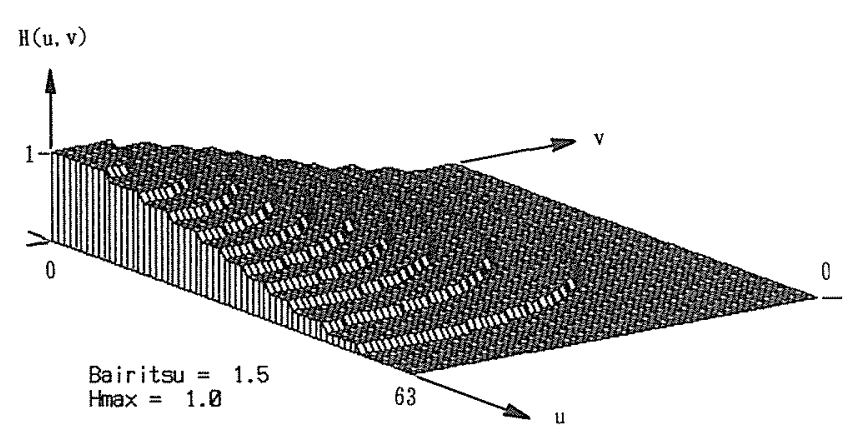

(a) $k 1=k 2=1$

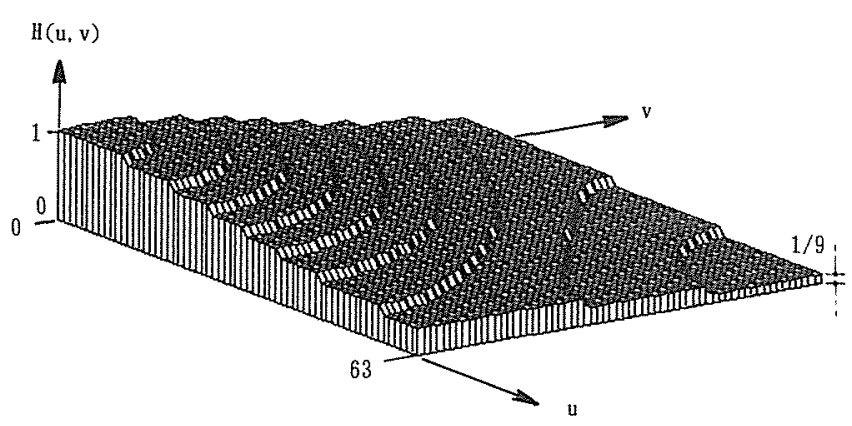

(b) $\mathrm{k} 1=\mathrm{k} 2=2$

Fig. 4 3-D representation of a function of two variables: $H(u, v)$ with characteristics of spatial low-pass filter $k 2=2$. This function shows a concentric type as regards the division of the spatial frequency domain in contrast to Fig. 3, and shows the characteristics of the spatial lowpass filter.

It is difficult to completely decide the process of division of spatial frequency domain and the combination of an optimum threshold for modifying transform coefficients obtained by the DCT. In this paper, the division method of the type $\langle\mathrm{C}\rangle$ in Fig. 3, i.e., only half of the spatial frequency domain is used with a view to performing an efficient compression of image data.

\section{Fidelity Criteria of Digital Images}

For color images with moderately complex scenes, all DCT-based modes of operation typically produce the following levels of picture quality. These levels are only a guideline. Note that the picture quality and the degree of compression can vary significantly according to an original image characteristics. The unit "bits/pixel" is often used for evaluations of fidelity. This unit means the total number of bits in the compressed image including the chrominance components divided by the number of samples in the gray level or luminance component.

Table 1 shows a typical bit-rate measure and its subjective aspects recommended by JPEG ${ }^{5}$. Although the calculation of bit-rate is very troublesome in connection with the process of transform coefficients and its coding techniques, it seems impractical for evaluation of image fidelity in general.

In this study, the difference image representation and fidelity measure are mainly used in order to demonstrate the image quality between the original image and the reconstructed image. Two general classes of criteria are sometimes used as the basis for such an assessment: (a) visual (or subjective) fidelity criteria and (b) quantitative (or objective) fidelity criteria. Although the quantitative

\begin{tabular}{|c|c|c|}
\hline & $\begin{array}{l}\text { 3it-rate } \\
\text { its/pixel] }\end{array}$ & Subjective aspects \\
\hline (A) & $0.25-0.5$ & $\begin{array}{l}\text { moderate to good quality. } \\
\text { sufficient for some applications }\end{array}$ \\
\hline (B) & $0.5-0.75$ & $\begin{array}{l}\text { good to very good quality. } \\
\text { sufficient for many applications }\end{array}$ \\
\hline (C) & $0.75-1.5$ & $\begin{array}{l}\text { excellent quality. } \\
\text { sufficient for most applications }\end{array}$ \\
\hline (D) & $1.5-2.0$ & $\begin{array}{l}\text { usually indistinguishable from the } \\
\text { original. } \\
\text { sufficient for the most deamding } \\
\text { applications }\end{array}$ \\
\hline
\end{tabular}
fidelity criteria offer a simple and convenient mechanism

Table 1 Bit-rate measures and its subjective aspects 
for evaluating information loss, most reconstructed (or decompressed) images ultimately are viewed by human beings. Consequently, measuring the image quality or fidelity by the visual evaluations of an observer is more appropriate and useful. In this case, the evaluations may be made using an absolute rating scale or by means of side-by-side comparison of two images. But, it is pointed out that the results of visual fidelity criteria are not always in accord with that of quantitative fidelity criteria.

When the degree of data loss can be expressed as a function of the original or input image and the compressed or reconstructed output image, it is said to be based on an objective fidelity criterion.

The root-mean-square-error: Erms between $x(m, n)$ and $\hat{x}(m, n)$ is defined as the square root of the squared error averaged over $\mathrm{M} \times \mathrm{N}$ array.

$$
\text { Erms }=\left\{(1 / M N) \sum_{m} \sum_{n}[x(m, n)-\hat{x}(m, n)]^{2}\right\}^{1 / 2}
$$

where, $x(m, n)$ : original image data $\hat{x}(m, n)$ : reconstructed or decompressed image data

A closely related objective fidelity criterion is known as the mean-square signal-to-noise ratio of the original and reconstructed image data

$$
\mathrm{SNR}=\frac{\sum_{\mathrm{m}} \sum_{\mathrm{n}} \hat{\mathrm{x}}(\mathrm{m}, \mathrm{n})^{2}}{\sum_{\mathrm{m}} \sum_{\mathrm{n}}[\mathrm{x}(\mathrm{m}, \mathrm{n})-\hat{\mathrm{x}}(\mathrm{m}, \mathrm{n})]^{2}}
$$

The mean-square error expression in Eq. (5) is often called the normalized least-square error, and is defined by a different expression as follows. This expression is popular because it correlates reasonably with subjective visual quality estimation and is mathematically tractable.

$$
E_{\text {NLSE }}=\frac{\sum_{m} \sum_{n}|x(m, n)-\hat{x}(m, n)|^{2}}{\sum_{m} \sum_{n}|x(m, n)|^{2}}
$$

Table 2 shows the typical fidelity measures for evaluate the difference between two similar or different digital image data ${ }^{6}$. Note that [1] MSE and [3] MAE are equivalent to [2] NMSE and [4] NMAE in view of the value of denominator, respectively. The correlation coefficient $\rho$ is very available in place of [5] ncc for evaluating the degree of two similar digital image data. The SNR defined by Eq. (6) is a little different from the NMSE formulated in Table 2 as for the numerator and denominator, respectively. On the other hand, the ENSLE defined by Eq. (7) is equal to the NMSE shown in Table 2.

\section{Visual Appearance and Its Fidelity of Computer- Displayed Images}

The original digital image data are composed of $64 \times 64$ pixels with 32 grey-levels, and may be used as the simple test data for many image processing techniques and their applications ${ }^{7)}$.

Note: Figs. 5, 7, 8 and 9 are on the front pages.

Table 2 Computation Formulas for Statistical Measures Between Two Image Data*

$$
\begin{aligned}
& \text { [1] Hean Square Error (M S E) } \\
& \begin{aligned}
M S E & =E\left\{[x(m, n)-\hat{x}(m, n)]^{2}\right\} \\
& =\frac{1}{M N} \sum_{m=0}^{M-1} \sum_{m=0}^{N-1}[x(m, n)-\hat{x}(m, n)]^{2}
\end{aligned}
\end{aligned}
$$

[2] Normalized Hean Square Error (NM S E)

$$
\begin{gathered}
\text { NMSE }=\frac{\text { MSE }}{\frac{1}{M N} \sum_{m=1}^{M-1} \sum_{n=1}^{N-1}[x(m, n)]^{2}} \\
\text { SNR }=-10 \log _{10} \text { (NMSE) [dB] }
\end{gathered}
$$

[3] Hean Absolute Error (M E )

$$
\text { MAE }=\frac{1}{M N} \sum_{m=0}^{M-1} \sum_{n=0}^{N-1}|x(m, n)-\hat{x}(m, n)|
$$

[4] Normalized Hean Absolute Error (NMAE)

$$
\text { NMAE }=\frac{\text { MAE }}{\frac{1}{M N} \sum_{m=0}^{M-1} \sum_{m=0}^{N-1}|x(m, n)|}
$$

[5] Normalized Correlation Coefficient ( $\mathrm{n} \mathrm{c} \mathrm{c}$ )

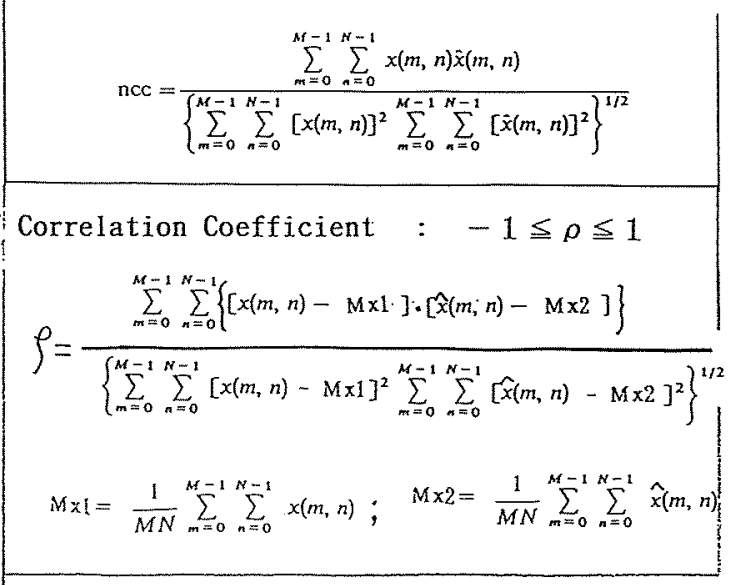

* K.R. Rao and P. Yip: [Discrete Cosine Transform Algorithms, Advantages, Applications], Academic Press, Inc. (1990) 
Fig. 5 shows an example of still image displayed using 16 levels in quasi-color and in monochrome color. The visual impressions of computer-displayed images are affected by not only the display techniques such as half- tone or quasi-color representation, but the size of image, the number of pixel resolution, the number of gray levels, etc.

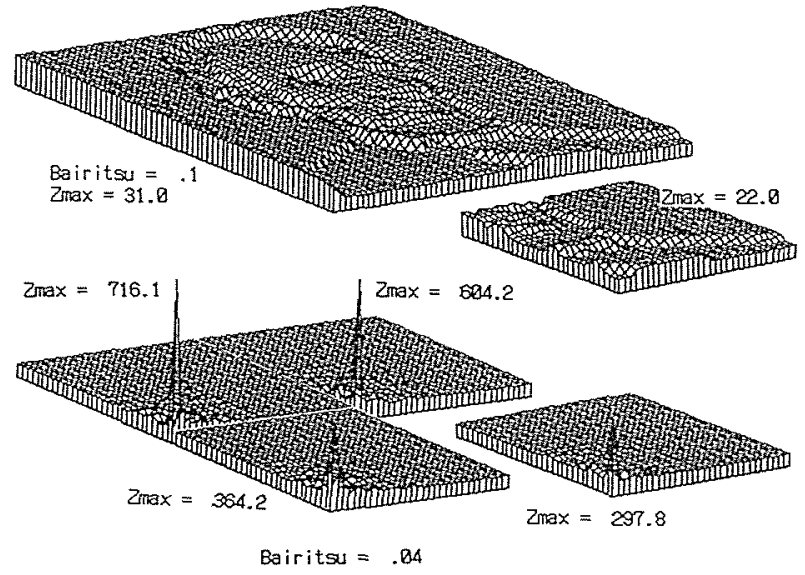

Bairitsu $=.04$

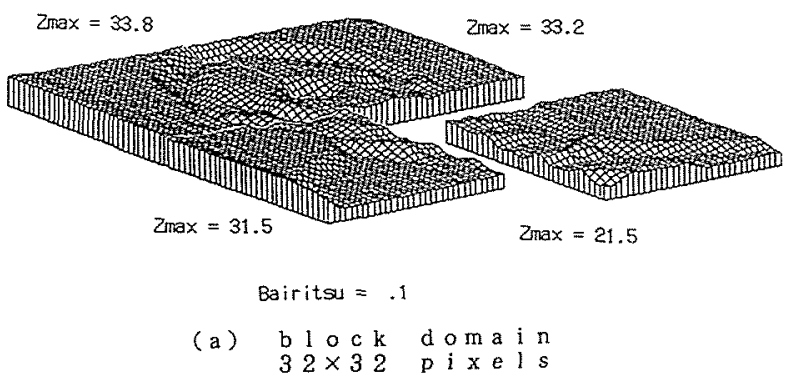

15.8
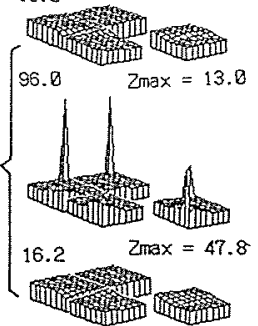

$z_{\max }=14.9$

(c) b lock doma i $8 \times 8$ p i x e I s

Fig. 6 3-D representations of original image, its absolute value of cosine transformed coefficients, and reconstructed image

Fig. 6 demonstrates a 3-D representation of the original image, its absolute value of cosine transformed coefficients, and the reconstructed image by using the original monochrome image data with 32 grey-levels and 64 $\times 64$ pixels. A set of $32 \times 32,16 \times 16$ and $8 \times 8$ small block pixels is used. A part of lower right domain is displayed separately for the sake of a discrimination of block distortion. It is possible to find out intuitively the effect of block distortion in some degree. Note that the $1 / 4$ domain of the adaptive spatial filter shown in Fig. $3<\mathrm{B}\rangle$ is used as the discrete cosine transform (DCT) coefficients. The extent of the concentration of energy in the domain of spatial frequency is discriminate from the results of 3 D representations.

Fig. 7 shows the quasi-color representation of the original and reconstructed images, and the difference image composed of four kinds of block pixels, respec- tively. The only 16 quasi-color levels are used for display the results. The two combinations of threshold values: Th1 and Th2 are specified for the adaptive spatial filter shown in Fig. $3<\mathrm{C}>$. As a result, the about $1 / 2$ domain of cosine transform coefficients which contain DC and low spatial frequency component is used in order to display the reconstructed and difference images. The difference image is defined as $|x(m, n)-X(m, n)|$ and is demonstrated in quasi-color.

It should be noted that a part of uniform and black domain demonstrates the accordance of the original and reconstructed images with regard to the display results in the difference image. The horizontal and vertical lines are depicted in order to discern the domain of each block pixel.

Fig. 8 shows the quasi-color representations of the original and reconstructed images, and the difference 
image composed of $16 \times 16$ block pixels, respectively. The two kinds of threshold values: Th $1=7$ and Th $2=15$ are specified for the adaptive spatial filter shown in Fig. 3 $<\mathrm{C}>$.

As a means for intuitively understanding the aspect of block distortion or degradation of a reconstructed image in contrast to an original image, a display technique based on the difference image between the original and reconstructed images is used extensively. As a result, the degree of similarity or difference between the two may be simply checked irrespective of various numerical evaluation measures. The quasi-color representation of the difference image is very useful for the intuitive evaluation of image quality, i.e., image fidelity.

Fig. 9 shows the difference image composed of $16 \times 16$ block pixels in quasi-color. In this figure, the number of quasi-color levels is altered from 16 to 4 in order to demonstrate the intuitive difference between the original image and reconstructed image. A part of the black domain increases taking it all in all, and the border line of original image is stressed in contrast to the results of Fig. 7 and Fig. 8.

Fig. 10 shows a relation between fidelity measures defined by Table 2 and block division on condition that the size of block pixel is set to $32 \times 32$. The combination of four kinds of threshold values is specified in advance for the adaptive spatial filter shown in Fig. $3<\mathrm{C}>$.

The expression of mean square error (MSE) is different from that of mean absolute error (MAE), but the two expressions have the almost same characteristics as regards the fidelity evaluation. The expression of normalized mean square error (NMAE) is equivalent to that of normalized mean absolute error (NMAE) as regards the characteristics of fidelity evaluation.

Note that there are discernible differences between the two in the interval of dynamic range, i.e., numerical values.

Fig. 11 shows a relation between fidelity measures, i.e., NMSE and SNR and the size of block pixels. The dynamic range of SNR is pretty limited in comparison with the results of NMSE in the case of two kinds of image data. Note that the computation formulas of Table 2 are used, and only the value of maximum-minimum for each fidelity measure are demonstrated in this figure. If the two digital image data are similar to each other, the value of NMSE approaches to zero. On the contrary, the value of SNR becomes large gradually. It is difficult to describe the standard criterion, because the values are affected by a variety of images, its size of block pixel etc.

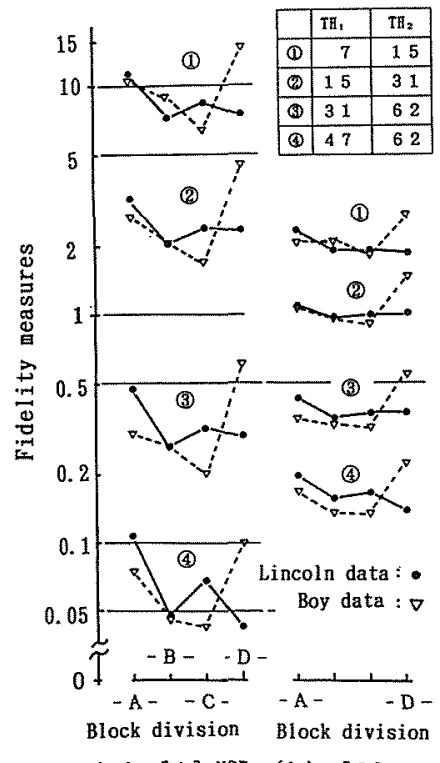

(a) [1] USE (b) [3] $\mathrm{HAE}$

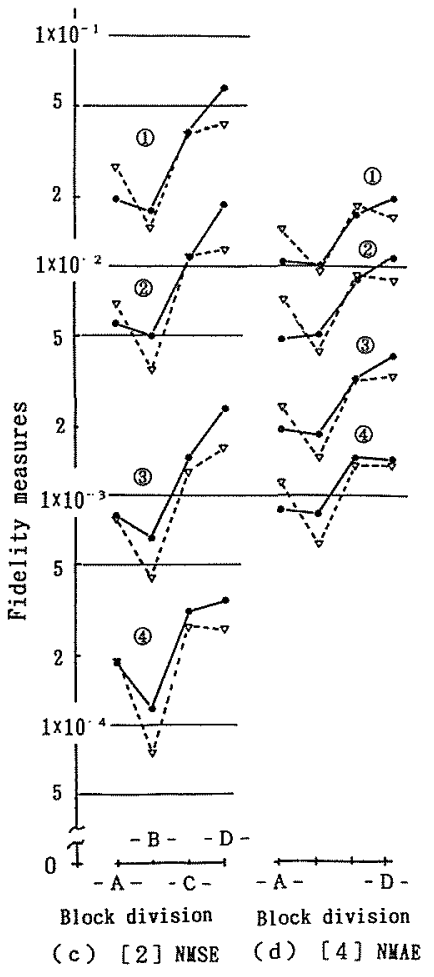

Fig. 10 Relation between fidelity measures and block division ( $32 \times 32$ block pixels) in the case of DCT

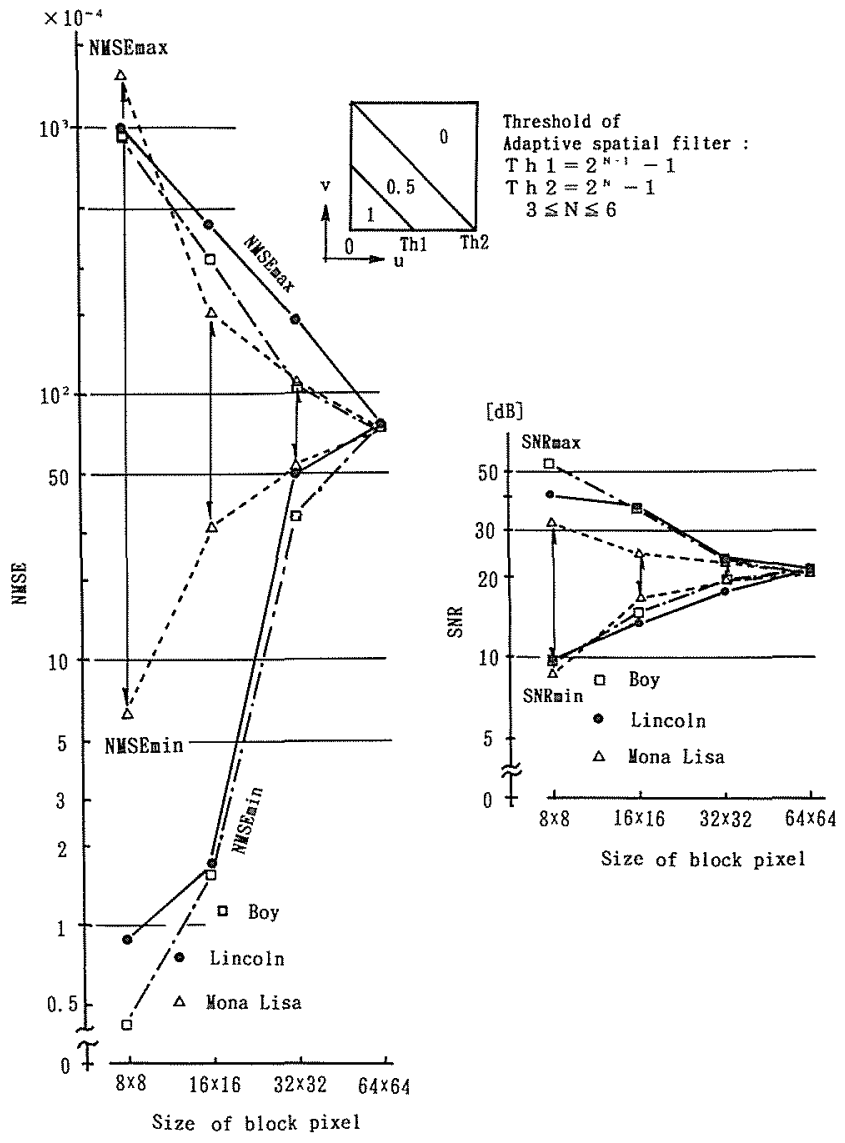

Fig. 11 Relation between fidelity measures: NMSE ; SNR and size of block pixel 
Fig. 12 shows a relation between fidelity measure, i.e., coefficient of correlation and the sequential No. of block pixel in the case of two kinds of images: Lincoln data and Mona Lisa data. Moreover, the average value for the two image data: $\rho_{\mathrm{M} \text {. AV }}$ and $\rho_{\mathrm{L} \text {. AV }}$ are demonstrated on the same figure. The result of the right side corresponds to
In this paper, only the results of quantitative evaluation between the original image and the reconstructed image are examined and discussed, because an objective affect of the distorted image and block distortion is demonstrated using 3-D and/or monochrome and quasi-color representation.
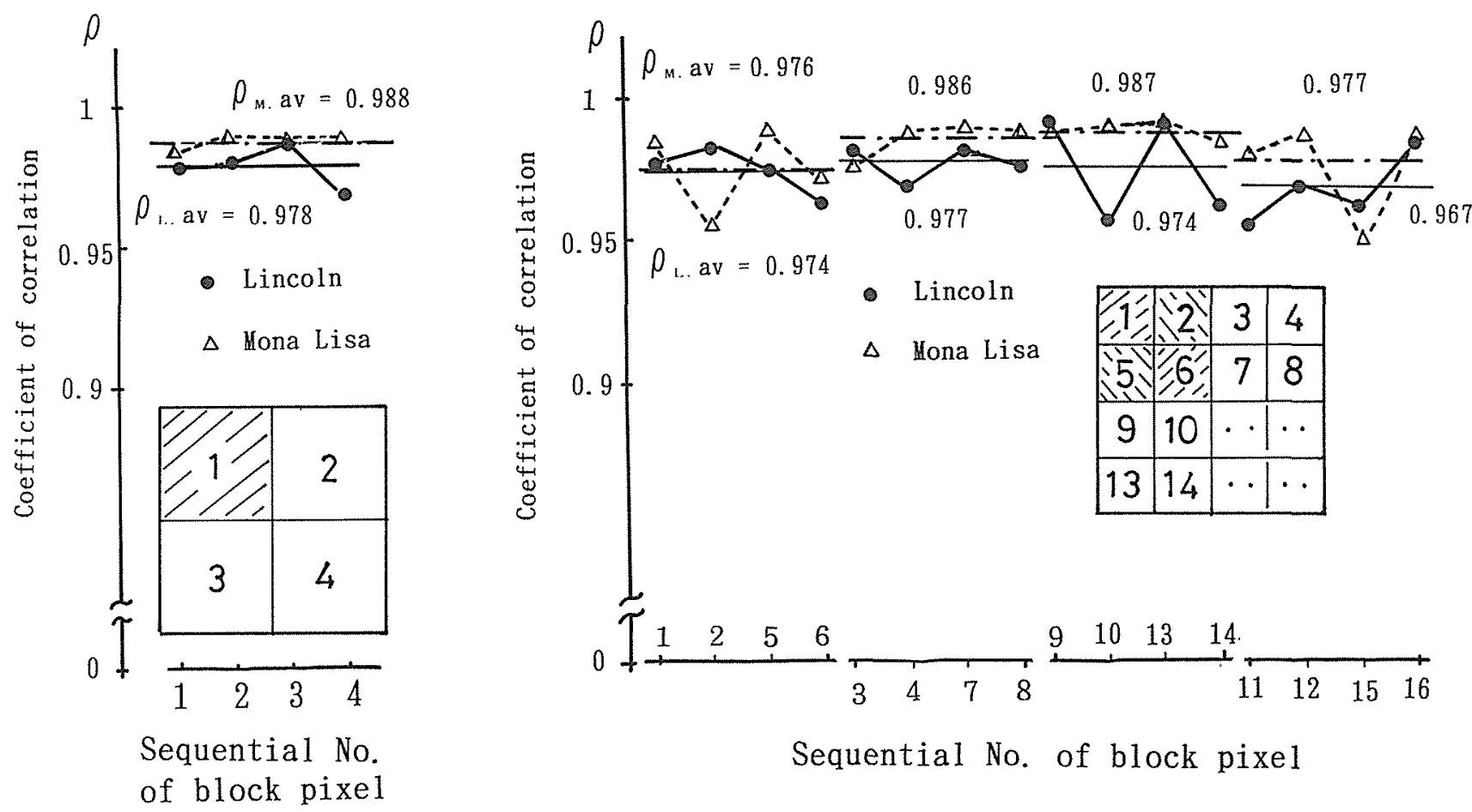

Fig. 12 Relation between fidelity measure: coefficient of correlation and sequential No. domain of block pixel

Fig. 8 , because the size of block pixel is composed of 16 $\times 16$ block pixels, and the same threshold values are specified as Th1 $=7$ and Th $2=15$. Note that the computation formula of Table 2 is used for discerning the degree of similarity between the original image data and the reconstructed image data.

Fig. 13 as well as Fig. 12 shows a relation between fidelity measure, i.e., coefficient of correlation and the sequential No. of block pixel in the case of two kinds of images. In this figure, the $8 \times 8$ block pixels are used, and correspond to the lower results of Fig. 7.

The subjective quality of a digital image can be generally evaluated by showing the image many observers, and by averaging the results of their evaluations. On the other hand, the statistical measures are sometimes called fidelity measures. Various computation formulas for the statistical measures are often employed in order to compute the quantitative values between the two different or similar images. There is a difference between the visual impressions of reconstructed image and the results of statistical evaluation measures.

\section{Conclusions}

The fidelity measures for evaluating image quality were calculated using the NMSE or SNR, and coefficient of correlation in connection with the size of block pixel, and the following results were obtained.

(1) It is possible to carry out the intuitive estimation between an original image and a reconstructed image by means of 3-D and/or quasi-color representation. The discontinuity of border among each block pixel is a little discernible in the case of 3-D representation as the size of block pixel becomes small.

(2) Original image data are effectively compressed using the DCT and the concept of adaptive spatial filter, and the reconstructed images with good fidelity are demonstrated by taking account of the appropriate threshold values for Th1 and Th2.

(3) The results of the difference image displayed in quasi-color are effective for the visual evaluation of image quality such as block distortion or dis- 

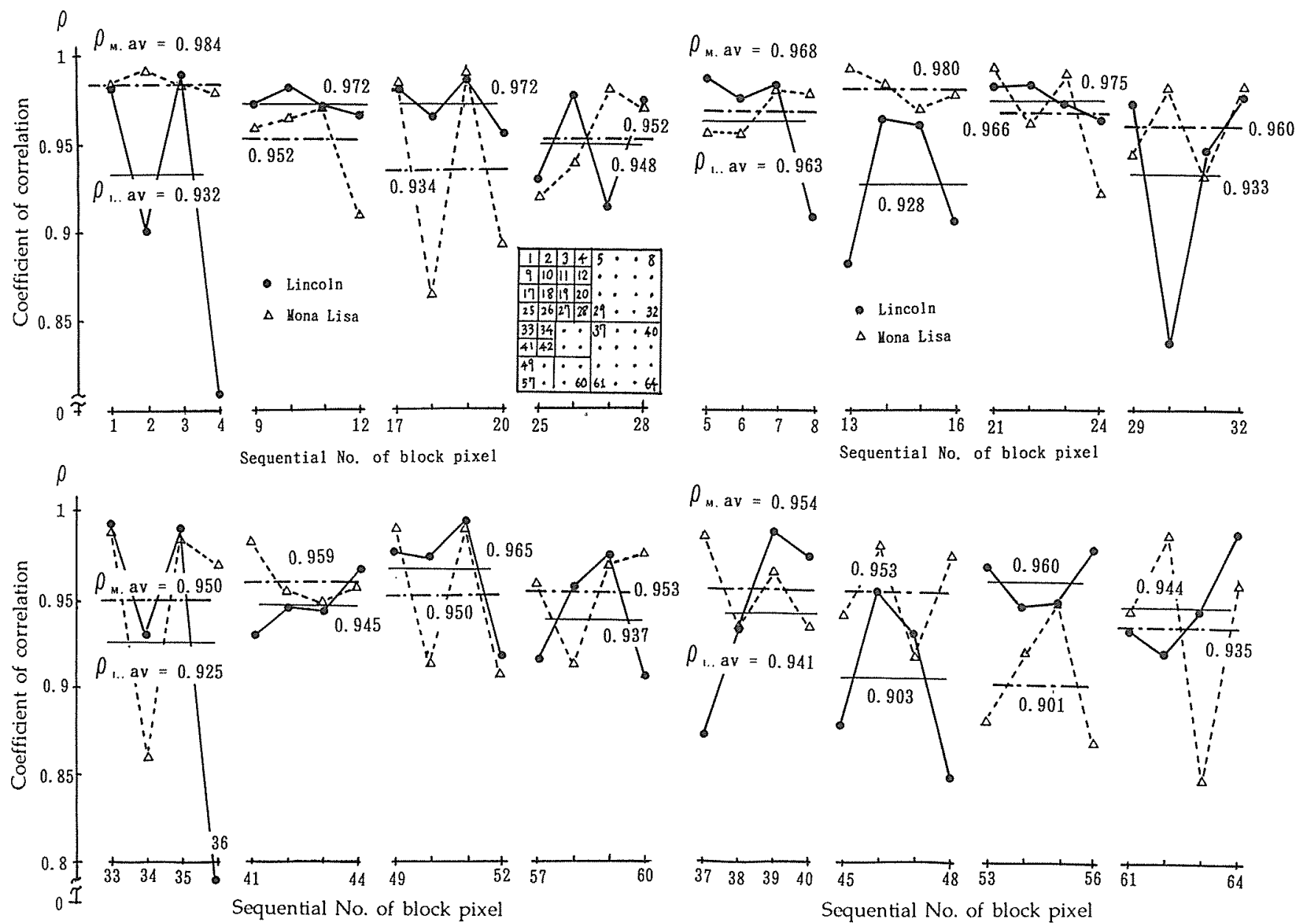

$\rho_{M, \text { av }}=0.954$
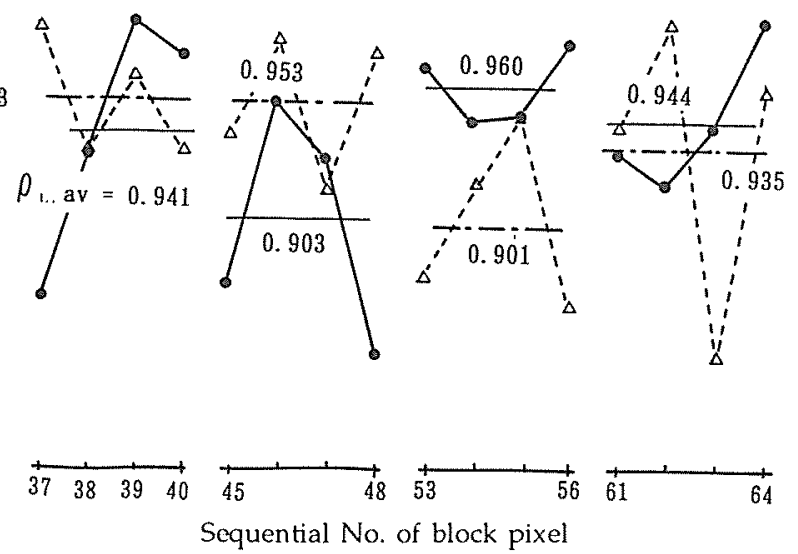

Fig. 13 Relation between fidelity measure: coefficient of correlation and sequential No. domain of block pixel in the case of $8 \times 8$ block pixels

torted images, on condition that the total number of quasi-color levels is altered from 16 to 4 including block.

\section{References}

(1) M. Rabbani and P.W. Jones: Digital Image Compression Technique, SPIE Opt. En. Press (1991)

B.C. Smith and L.A. Rowe: Algorithms for Manipulating Compressed Images, IEEE Computer Graphics \& Applications, Vol. 13, No. 5 (1993) pp. 34-42

(2) International Standard DIS 10918-1; CCITT Recommendation T.81: Information Technology - Digital Compression and Coding of Continuous-Tone Still Images, (1991)

(3) R.J. Clarke: Transform Coding of Images, Academic Press, (1985)

(4) W.B. Pennebaker and J.L. Mitchell: JPEG Still Image Data Compression Standard, VNR (1993)
(5) G.K. Wallance: The JPEG Still Picture Compression Standard, Communications of the ACM, Vol. 34, No. 4 (1991) pp. 30-44

(6) K.R. Rao and P. Yip: Discrete Cosine Transform Algorithm, Advantages, Applications, Academic Press, Inc. (1990)

(7) R.C. Gonzalez and P. Wints: Digital Image Processing Addison-Wesley Pub. Co. (1987)

R. C. Gonzalez and R.E. Woods: Digital Image Processing Addison-Wesley Pub. Co. (1992)

\section{Appendix}

Linear color transformations based on reference (4)

For the three primary colors: R (red), G (green) and B (blue), the luminance $Y$ of any color can be calculated from the following weighted sum.

$Y=0.3 R+0.6 G+0.1 B$ 
The term chrominance is defined as the difference between a color and a reference white at the same luminance. A set of color differences: $U$ and $V$ may be defined as follows.

$$
\mathrm{U}=\mathrm{B}-\mathrm{Y} \text { and } \mathrm{V}=\mathrm{R}-\mathrm{Y}
$$

Another color space: YIQ is used in the North American television systems. The YIQ color space is related to the YUV color space. I and $Q$ are related only to $U$ and V.

$$
\mathrm{I}=0.74 \mathrm{~V}-0.27 \mathrm{U} \text { and } \mathrm{Q}=0.48 \mathrm{~V}+0.41 \mathrm{U}
$$

Still another color space: $\mathrm{YCbCr}$ is used extensively in the development of the JPEG standard. $U$ and $V$ are scaled and zero-shifted to produce a set of two variables: $\mathrm{CbCr}$.

$$
\mathrm{Cb}=(\mathrm{U} / 2)+0.5 \text { and } \mathrm{Cr}=(\mathrm{V} / 1.6)+0.5
$$

